\title{
Common Fixed Point for Three Pairs of Self-Maps Satisfying Common (E.A) Property in Generalized Metric Spaces
}

\author{
Feng Gu and Yun Yin \\ Institute of Applied Mathematics and Department of Mathematics, Hangzhou Normal University, Hangzhou, Zhejiang 310036, China
}

Correspondence should be addressed to Feng Gu; gufeng99@sohu.com

Received 1 February 2013; Accepted 7 February 2013

Academic Editor: Yisheng Song

Copyright (C) 2013 F. Gu and Y. Yin. This is an open access article distributed under the Creative Commons Attribution License, which permits unrestricted use, distribution, and reproduction in any medium, provided the original work is properly cited.

Using the concept of common (E.A) property, we prove a common fixed point theorem for three pairs of weakly compatible self-maps satisfying a new contractive condition in the framework of a generalized metric space. Our results do not rely on any commuting or continuity condition of mappings. An example is provided to support our result. The results obtained in this paper differ from the recent relative results in the literature.

\section{Introduction}

The study of fixed points and common fixed points of mappings satisfying certain contractive conditions has been at the center of rigorous research activity. In 2006, Mustafa and Sims [1] introduced the concept of generalized metric spaces or simply $G$-metric spaces as a generalization of the notion of a metric space. Based on the notion of generalized metric spaces, Mustafa et al. [2-5], Obiedat and Mustafa [6], Aydi et al. [7, 8], Gajié and Stojakovié [9], and Zhou and $\mathrm{Gu}[10]$ obtained some fixed point results for mappings satisfying different contractive conditions. Shatanawi [11] obtained some fixed point results for $\Phi$-maps in $G$-metric spaces. Chugh et al. [12] obtained some fixed point results for maps satisfying property $P$ in $G$-metric spaces. Study of common fixed point problems in $G$-metric spaces was initiated by Abbas and Rhoades [13]. Subsequently, many authors obtained many common fixed point theorems for the mappings satisfying different contractive conditions (see [1432] for more details). Recently, Abbas et al. [33] and Mustafa et al. [34] obtained some common fixed point results for a pair of mappings satisfying (E.A) property under certain generalized strict contractive conditions in $G$-metric spaces. Long et al. [35] obtained some common coincidence and common fixed points results of two pairs of mappings when only one pair satisfies (E.A) property in the framework of a generalized metric space.
The aim of this paper is to study common fixed point of three pairs of mappings for which only two pairs need to satisfy common (E.A) property in the framework of $G$ metric spaces. Our results do not rely on any commuting or continuity condition of mappings.

\section{Definitions and Preliminary Results}

In this section, we present the necessary definitions and results in $G$-metric spaces.

Definition 1 (see [1]). Let $X$ be a nonempty set, and let $G: X \times$ $X \times X \rightarrow R^{+}$be a function satisfying the following axioms:

(G1) $G(x, y, z)=0$ if $x=y=z$;

(G2) $0<G(x, x, y)$, for all $x, y \in X$ with $x \neq y$;

(G3) $G(x, x, y) \leq G(x, y, z)$, for all $x, y$, and $z \in X$ with $z \neq \mathrm{y}$

(G4) $G(x, y, z)=G(x, z, y)=G(y, z, x)=\cdots$ (symmetry in all three variables);

(G5) $G(x, y, z) \leq G(x, a, a)+G(a, y, z)$ for all $x, y, z$, and $a \in X$, (rectangle inequality).

Then the function $G$ is called a generalized metric, or more specifically a $G$-metric on $X$ and the pair $(X, G)$ are called a $G$-metric space. 
It is known that the function $G(x, y$, and $z)$ on $G$-metric space $X$ is jointly continuous in all three of its variables, and $G(x, y, z)=0$ if and only if $x=y=z$ (see [1] for more details and the reference therein).

Definition 2 (see $[1])$. Let $(X, G)$ be a $G$-metric space, and let $\left\{x_{n}\right\}$ be a sequence of points in $X$; a point $x$ in $X$ is said to be the limit of the sequence $\left\{x_{n}\right\}$ if $\lim _{m, n \rightarrow \infty} G\left(x, x_{n}, x_{m}\right)=0$, and one says that sequence $\left\{x_{n}\right\}$ is $G$-convergent to $x$.

Thus, if $x_{n} \rightarrow x$ in a $G$-metric space $(X, G)$, then for any $\epsilon>0$ there exists $N \in \mathbb{N}$ (throughout this paper we mean by $\mathbb{N}$ the set of all natural numbers) such that $G\left(x, x_{n}\right.$, and $\left.x_{m}\right)<\epsilon$, for all $n, m \geq N$.

Proposition 3 (see [1]). Let $(X, G)$ be a $G$-metric space; then the followings are equivalent:

(1) $\left\{x_{n}\right\}$ is G-convergent to $x$.

(2) $G\left(x_{n}, x_{n}, x\right) \rightarrow 0$ as $n \rightarrow \infty$.

(3) $G\left(x_{n}, x, x\right) \rightarrow 0$ as $n \rightarrow \infty$.

(4) $G\left(x_{n}, x_{m}, x\right) \rightarrow 0$ as $n, m \rightarrow \infty$.

Definition 4 (see [1]). Let $(X, G)$ be a $G$-metric space. A sequence $\left\{x_{n}\right\}$ is called $G$-Cauchy sequence if, for each $\epsilon>$ 0 , there exists $N \in \mathbb{N}$ such that $G\left(x_{n}, x_{m}\right.$, and $\left.x_{l}\right)<\epsilon$ for all $n, m$, and $l \geq N$, that is, if $G\left(x_{n}, x_{m}, x_{l}\right) \rightarrow 0$ as $n, m$, and $l \rightarrow \infty$.

Definition 5 (see [1]). A $G$-metric space $(X, G)$ is said to be $G$ complete (or a complete $G$-metric space) if every $G$-Cauchy sequence in $(X, G)$ is $G$-convergent in $X$.

Proposition 6 (see $[1])$. Let $(X, G)$ be a $G$-metric space. Then the following are equivalent.

(1) The sequence $\left\{x_{n}\right\}$ is G-Cauchy.

(2) For every $\epsilon>0$, there exists $k \in \mathbb{N}$ such that $G\left(x_{n}, x_{m}, x_{m}\right)<\epsilon$, for all $n, m \geq k$.

Proposition 7 (see $[1])$. Let $(X, G)$ be a $G$-metric space. Then the function $G(x, y$, and $z)$ is jointly continuous in all three of its variables.

Definition 8 (see [36]). Let $f$ and $g$ be self-maps of a set $X$. If $w=f x=g x$ for some $x$ in $X$, then $x$ is called a coincidence point of $f$ and $g$, and $w$ is called point of coincidence of $f$ and $g$.

Definition 9 (see [36]). Two self-mappings $f$ and $g$ on $X$ are said to be weakly compatible if they commute at coincidence points.

Definition 10 (see [33]). Let $X$ be a $G$-metric space. Self-maps $f$ and $g$ on $X$ are said to satisfy the $G$-(E.A) property if there exists a sequence $\left\{x_{n}\right\}$ in $X$ such that $\left\{f x_{n}\right\}$ and $\left\{g x_{n}\right\}$ are $G$ convergent to some $t \in X$.

Definition 11. Let $(X, d)$ be a $G$-metric space and $A, B, S$, and $T$ four self-maps on $X$. The pairs $(A, S)$ and $(B, T)$ are said to satisfy common (E.A) property if there exist two sequences $\left\{x_{n}\right\}$ and $\left\{y_{n}\right\}$ in $X$ such that $\lim _{n \rightarrow \infty} A x_{n}=\lim _{n \rightarrow \infty} S x_{n}=$ $\lim _{n \rightarrow \infty} B y_{n}=\lim _{n \rightarrow \infty} T y_{n}=t$, for some $t \in X$.

Definition 12 (see [17]). Self-mappings $f$ and $g$ of a $G$-metric space $(X, G)$ are said to be compatible if $\lim _{n \rightarrow \infty}$ $G\left(f g x_{n}, g f x_{n}, g f x_{n}\right)=0$ and $\lim _{n \rightarrow \infty} G\left(g f x_{n}, f g x_{n}, f g x_{n}\right)=$ 0 , whenever $\left\{x_{n}\right\}$ is a sequence in $X$ such that $\lim _{n \rightarrow \infty} f x_{n}$ $=\lim _{n \rightarrow \infty} g x_{n}=t$, for some $t \in X$.

Definition 13 (see [16]). A pair of self-mappings $(f, g)$ of a $G$ metric space is said to be weakly commuting if

$$
G(f g x, g f x, g f x) \leq G(f x, g x, g x), \quad \forall x \in X .
$$

Definition 14 (see [16]). A pair of self-mappings $(f, g)$ of a $G$ metric space is said to be $R$-weakly commuting, if there exists some positive real number $R$ such that

$$
G(f g x, g f x, g f x) \leq R G(f x, g x, g x), \quad \forall x \in X .
$$

\section{Main Results}

In this section, we obtain some unique common fixed point results for six mappings satisfying certain generalized contractive conditions in the framework of a generalized metric space. We start with the following result.

Theorem 15. Let $(X, G)$ be a G-metric space. Suppose mappings $f, g, h, R, S$, and $T: X \rightarrow X$ satisfying the following conditions:

$$
\begin{aligned}
& G(f x, g y, h z) \\
& \leq \phi(\max \{ G(R x, S y, T z), G(f x, R x, R x), \\
& G(g y, S y, S y), G(h z, T z, T z), \\
& \frac{1}{3}[G(f x, S y, T z)+G(R x, g y, T z) \\
&+G(R x, S y, h z)], \\
& \frac{1}{3}[G(f x, g y, T z)+G(f x, S y, h z) \\
&+G(R x, g y, h z)]\}),
\end{aligned}
$$

for all $x, y$, and $z \in X$, where $\phi:[0, \infty) \rightarrow[0, \infty)$ is the function satisfying $0<\phi(t)<t$, for all $t>0$. If one of the following conditions is satisfied, then the pairs $(f, R),(g, S)$, and $(h, T)$ have a common point of coincidence in $X$ :

(i) the subspace $R X$ is closed in $X, f X \subseteq S X, g X \subseteq T X$, and two pairs of $(f, R)$ and $(g, S)$ satisfy common (E.A) property;

(ii) the subspace $S X$ is closed in $X, g X \subseteq T X, h X \subseteq R X$, and two pairs of $(g, S)$ and $(h, T)$ satisfy common (E.A) property;

(iii) the subspace $T X$ is closed in $X, f X \subseteq S X, h X \subseteq R X$, and two pairs of $(f, R)$ and $(h, T)$ satisfy common (E.A) property. 
Moreover, if the pairs $(f, R),(g, S)$, and $(h, T)$ are weakly compatible, then $f, g, h, R, S$, and $T$ have a unique common fixed point in $X$.

Proof. First, we suppose that the subspace $R X$ is closed in $X$, $f X \subseteq S X, g X \subseteq T X$, and two pairs of $(f, R)$ and $(g, S)$ satisfy common (E.A) property. Then by Definition 11 we know that, there exist two sequences $\left\{x_{n}\right\}$ and $\left\{y_{n}\right\}$ in $X$ such that

$$
\lim _{n \rightarrow \infty} f x_{n}=\lim _{n \rightarrow \infty} R x_{n}=\lim _{n \rightarrow \infty} g y_{n}=\lim _{n \rightarrow \infty} S y_{n}=t
$$

for some $t \in X$.

Since $g X \subseteq T X$, there exists a sequence $\left\{z_{n}\right\}$ in $X$ such that $g y_{n}=T z_{n}$. Hence $\lim _{n \rightarrow \infty} T z_{n}=t$. Next, we will show $\lim _{n \rightarrow \infty} h z_{n}=t$. In fact, if $\lim _{n \rightarrow \infty} h z_{n} \neq t$, then from condition (3), we can get

$$
\begin{gathered}
G\left(f x_{n}, g y_{n}, h z_{n}\right) \\
\leq \phi\left(\operatorname { m a x } \left\{G\left(R x_{n}, S y_{n}, T z_{n}\right), G\left(f x_{n}, R x_{n}, R x_{n}\right),\right.\right. \\
G\left(g y_{n}, S y_{n}, S y_{n}\right), G\left(h z_{n}, T z_{n}, T z_{n}\right), \\
\frac{1}{3}\left[G\left(f x_{n}, S y_{n}, T z_{n}\right)+G\left(R x_{n}, g y_{n}, T z_{n}\right)\right. \\
\left.+G\left(R x_{n}, S y_{n}, h z_{n}\right)\right], \\
\frac{1}{3}\left[G\left(f x_{n}, g y_{n}, T z_{n}\right)+G\left(f x_{n}, S y_{n}, h z_{n}\right)\right. \\
\left.\left.\left.+G\left(R x_{n}, g y_{n}, h z_{n}\right)\right]\right\}\right) .
\end{gathered}
$$

On letting $n \rightarrow \infty$ and based on the property of $\phi$, we can obtain

$$
\begin{aligned}
G\left(t, t, \lim _{n \rightarrow \infty} h z_{n}\right) & \leq \phi\left(G\left(\lim _{n \rightarrow \infty} h z_{n}, t, t\right)\right) \\
& <G\left(\lim _{n \rightarrow \infty} h z_{n}, t, t\right) .
\end{aligned}
$$

It is contradiction; so $\lim _{n \rightarrow \infty} h z_{n}=t$.

Since $R X$ is a closed subspace of $X$ and $\lim _{n \rightarrow \infty} R x_{n}=t$, there exists a $p$ in $X$ such that $t=R p$. We claim that $f p=t$. Suppose not; then by using (3) we obtain

$$
\begin{gathered}
G\left(f p, g y_{n}, h z_{n}\right) \\
\leq \phi\left(\operatorname { m a x } \left\{G\left(R p, S y_{n}, T z_{n}\right), G(f p, R p, R p),\right.\right. \\
G\left(g y_{n}, S y_{n}, S y_{n}\right), G\left(h z_{n}, T z_{n}, T z_{n}\right), \\
\frac{1}{3}\left[G\left(f p, S y_{n}, T z_{n}\right)+G\left(R p, g y_{n}, T z_{n}\right)\right. \\
\left.+G\left(R p, S y_{n}, h z_{n}\right)\right], \\
\frac{1}{3}\left[G\left(f p, g y_{n}, T z_{n}\right)+G\left(f p, S y_{n}, h z_{n}\right)\right. \\
\left.\left.\left.+G\left(R p, g y_{n}, h z_{n}\right)\right]\right\}\right) .
\end{gathered}
$$

Taking $n \rightarrow \infty$ on the two sides of the above inequality and using the property of $\phi$, we can get

$$
G(f p, t, t) \leq \phi(\max \{G(f p, t, t)\})<G(f p, t, t) .
$$

Which is contradiction, and so $f p=t=R p$. Hence, $p$ is the coincidence point of pair $(f, R)$.

By the condition $f X \subseteq S X$ and $f p=t$, there exists a point $u$ in $X$ such that $t=S u$. Now, we claim that $g u=t$. In fact, if $g u \neq t$, then from (3) we have

$$
\begin{gathered}
G\left(f p, g u, h z_{n}\right) \\
\leq \phi\left(\operatorname { m a x } \left\{G\left(R p, S u, T z_{n}\right), G(f p, R p, R p),\right.\right. \\
G(g u, S u, S u), G\left(h z_{n}, T z_{n}, T z_{n}\right), \\
\frac{1}{3}\left[G\left(f p, S u, T z_{n}\right)+G\left(R p, g u, T z_{n}\right)\right. \\
\left.+G\left(R p, S u, h z_{n}\right)\right], \\
\frac{1}{3}\left[G\left(f p, g u, T z_{n}\right)+G\left(f p, S u, h z_{n}\right)\right. \\
\left.\left.\left.+G\left(R p, g u, h z_{n}\right)\right]\right\}\right) .
\end{gathered}
$$

Letting $n \rightarrow \infty$ on the two sides of the above inequality and using the property of $\phi$, we can obtain

$$
G(t, g u, t) \leq \phi(G(g u, t, t))<G(g u, t, t) .
$$

It is contradiction. Hence $g u=t=S t$, and so $u$ is the coincidence point of pair $(g, S)$.

Since $g X \subseteq T X$ and $g u=t$, there exists a point $v$ in $X$ such that $t=T v$. We claim that $h v=t$. If not, from (3) and the property of $\phi$, we have

$$
\begin{gathered}
G(t, t, h v) \\
=G(f p, g u, h v) \\
\leq \phi(\max \{G(R p, S u, T v), G(f p, R p, R p), \\
G(g u, S u, S u), G(h v, T v, T v), \\
\frac{1}{3}[G(f p, S u, T v)+G(R p, g u, T v) \\
+G(R p, S u, h v)], \\
\frac{1}{3}[G(f p, g u, T v)+G(f p, S u, h v) \\
+G(R p, g u, h v)]\}) \\
=\phi(G(h v, t, t)) \\
<G(h v, t, t) .
\end{gathered}
$$

It is contradiction. Hence $h v=t=T v$, and so $v$ is the coincidence point of pair $(h, T)$. 
Therefore, in all the above cases, we obtain $f p=R p=$ $g u=S u=h v=T v=t$. Now, weakly compatibility of the pairs $(f, R),(g, S)$, and $(h, T)$ gives that $f t=R t, g t=S t$, and $h t=T t$.

Next, we show that $f t=t$. In fact, if $f t \neq t$, then from (3) we have

$$
\begin{aligned}
& G(f t, t, t) \\
& =G(f t, g u, h v) \\
& \leq \phi(\max \{G(R t, S u, T v), G(f t, R t, R t), \\
& G(g u, S u, S u), G(h v, T v, T v), \\
& \frac{1}{3}[G(f t, S u, T v)+G(R t, g u, T v) \\
& +G(R t, S u, h v)], \\
& \frac{1}{3}[G(f t, g u, T v)+G(f t, S u, h v) \\
& +G(R t, g u, h v)]\}) \\
& =\phi(G(f t, t, t)) \\
& <G(f t, t, t)
\end{aligned}
$$

which is contradiction; hence $f t=t$, and so $f t=R t=t$. Similarly, it can be shown that $g t=S t=t$ and $h t=T t=t$; so we get $f t=g t=h t=R t=S t=T t=t$, which means that $t$ is a common fixed point of $f, g, h, R, S$, and $T$.

Next, we will show the common fixed point of $f, g, h$, $R, S$, and $T$ is unique. Actually, suppose that $w \in X ; w \neq t$ is another common fixed point of $f, g, h, R, S$, and $T$; then by condition (3) we have

$$
\begin{aligned}
& G(w, t, t) \\
& =G(f w, g t, h t) \\
& \leq \phi(\max \{G(R w, S t, T t), G(f w, R w, R w), \\
& G(g t, S t, S t), G(h t, T t, T t), \\
& \frac{1}{3}[G(f w, S t, T t)+G(R w, g t, T t) \\
& +G(R w, S t, h t)], \\
& \frac{1}{3}[G(f w, g t, T t)+G(f w, S t, h t) \\
& +G(R w, g t, h t)]\}) \\
& =\phi(G(w, t, t)) \\
& <G(w, t, t) .
\end{aligned}
$$

It is a contradiction, unless $w=t$; that is, mappings $f, g, h, R, S$, and $T$ have a unique common fixed point.
Finally, if condition (ii) or (iii) holds, then the argument is similar to that above; so we delete it.

This completes the proof of Theorem 15.

Now, we give an example to support Theorem 15.

Example 16. Let $X=[0,1]$ be a $G$-metric space with

$$
G(x, y, z)=|x-y|+|y-z|+|z-x| .
$$

We define mappings $f, g, h, R, S$, and $T$ on $X$ by

$$
\begin{aligned}
& f x=\left\{\begin{array}{ll}
1, & x \in\left[0, \frac{1}{2}\right] \\
\frac{5}{6}, & x \in\left(\frac{1}{2}, 1\right],
\end{array} \quad g x= \begin{cases}\frac{7}{8}, & x \in\left[0, \frac{1}{2}\right] \\
\frac{5}{6}, & x \in\left(\frac{1}{2}, 1\right],\end{cases} \right. \\
& h x=\left\{\begin{array}{ll}
\frac{6}{7}, & x \in\left[0, \frac{1}{2}\right] \\
\frac{5}{6}, & x \in\left(\frac{1}{2}, 1\right],
\end{array} \quad R x= \begin{cases}\frac{4}{5}, & x \in\left[0, \frac{1}{2}\right] \\
\frac{5}{6}, & x \in\left(\frac{1}{2}, 1\right) \\
\frac{6}{7}, & x=1,\end{cases} \right. \\
& S x=\left\{\begin{array}{ll}
1, & x \in\left[0, \frac{1}{2}\right] \\
\frac{5}{6}, & x \in\left(\frac{1}{2}, 1\right) \\
0, & x=1,
\end{array} \quad T x= \begin{cases}1, & x \in\left[0, \frac{1}{2}\right] \\
\frac{5}{6}, & x \in\left(\frac{1}{2}, 1\right) \\
\frac{7}{8}, & x=1 .\end{cases} \right.
\end{aligned}
$$

Note that $f, g, h, R, S$, and $T$ are discontinuous mappings. Clearly, the subspace $R X$ is closed in $X, f X \subseteq S X, g X \subseteq T X$, $h X \subseteq R X$, and the pairs $(f, R),(g, S)$, and $(h, T)$ are weakly compatible. Also, the pairs $(f, R)$ and $(g, S)$ satisfy common (E.A) property; indeed, $x_{n}=4 / 5$ and $y_{n}=3 / 4$ for each $n \in N$ are the required sequences. The control function $\phi:[0, \infty) \rightarrow[0, \infty)$ is defined by $\phi(t)=11 t / 12$.

First, we let

$$
\begin{gathered}
M(x, y, z) \\
=\max \{G(R x, S y, T z), G(f x, R x, R x), \\
G(g y, S y, S y), G(h z, T z, T z), \\
\frac{1}{3}[G(f x, S y, T z)+G(R x, g y, T z) \\
+G(R x, S y, h z)], \\
\frac{1}{3}[G(f x, g y, T z)+G(f x, S y, h z) \\
+G(R x, g y, h z)]\} .
\end{gathered}
$$

To prove (3), let us discuss the following cases.

Case 1. For $x, y$, and $z \in((1 / 2), 1]$, then we have $G(f x, g y, h z)=0$, and hence (3) is obviously satisfied. 
Case 2. For $x, y$, and $z \in[0,(1 / 2)]$, then we have

$$
\begin{gathered}
G(f x, g y, h z)=G\left(1, \frac{7}{8}, \frac{6}{7}\right)=\frac{2}{7}, \\
\phi(M(x, y, z)) \\
=\phi\left(\operatorname { m a x } \left\{G\left(\frac{4}{5}, 1,1\right), G\left(1, \frac{4}{5}, \frac{4}{5}\right), G\left(\frac{7}{8}, 1,1\right),\right.\right. \\
G\left(\frac{6}{7}, 1,1\right), \frac{1}{3}\left[G(1,1,1)+G\left(\frac{4}{5}, \frac{7}{8}, 1\right)\right. \\
\frac{1}{3}\left[G\left(1, \frac{7}{8}, 1\right)+G\left(1,1, \frac{6}{7}\right)\right. \\
=\phi\left(\max \left\{\frac{2}{5}, \frac{2}{5}, \frac{1}{4}, \frac{4}{15}, \frac{8}{35}\right\}\right) \\
=\phi\left(\frac{2}{5}\right)=\frac{11}{30} .
\end{gathered}
$$

Therefore,

$$
G(f x, g y, h z)=\frac{2}{7}<\frac{11}{30}=\phi(M(x, y, z)) .
$$

Case 3. For $x, y \in[0,(1 / 2)], z \in((1 / 2), 1]$, then we have

$$
G(f x, g y, h z)=G\left(1, \frac{7}{8}, \frac{5}{6}\right)=\frac{1}{3}
$$

Next we divide the study in two subcases.

(a) If $z \in((1 / 2), 1)$, then we have

$$
\begin{aligned}
\phi(M(x, y, z)) & \phi\left(\operatorname { m a x } \left\{G\left(\frac{4}{5}, 1, \frac{5}{6}\right), G\left(1, \frac{4}{5}, \frac{4}{5}\right), G\left(\frac{7}{8}, 1,1\right),\right.\right. \\
& G\left(\frac{5}{6}, \frac{5}{6}, \frac{5}{6}\right), \frac{1}{3}\left[G\left(1,1, \frac{5}{6}\right)+G\left(\frac{4}{5}, \frac{7}{8}, \frac{5}{6}\right)\right. \\
& \left.+G\left(\frac{4}{5}, 1, \frac{5}{6}\right)\right], \\
& \frac{1}{3}\left[G\left(1, \frac{7}{8}, \frac{5}{6}\right)+G\left(1,1, \frac{5}{6}\right)\right. \\
= & \phi\left(\max \left\{\frac{2}{5}, \frac{2}{5}, \frac{1}{4}, 0, \frac{53}{180}, \frac{49}{180}\right\}\right) \\
= & \phi\left(\frac{2}{5}\right)=\frac{11}{30} .
\end{aligned}
$$

(b) If $z=1$, then we get

$$
\begin{aligned}
\phi(M(x, y, z)) & \phi\left(\operatorname { m a x } \left\{G\left(\frac{4}{5}, 1, \frac{7}{8}\right), G\left(1, \frac{4}{5}, \frac{4}{5}\right), G\left(\frac{7}{8}, 1,1\right),\right.\right. \\
& G\left(\frac{5}{6}, \frac{7}{8}, \frac{7}{8}\right), \frac{1}{3}\left[G\left(1,1, \frac{7}{8}\right)+G\left(\frac{4}{5}, \frac{7}{8}, \frac{7}{8}\right)\right. \\
& \left.+G\left(\frac{4}{5}, 1, \frac{5}{6}\right)\right], \\
& \frac{1}{3}\left[G\left(1, \frac{7}{8}, \frac{7}{8}\right)+G\left(1,1, \frac{5}{6}\right)\right. \\
= & \phi\left(\max \left\{\frac{2}{5}, \frac{2}{5}, \frac{1}{4}, \frac{1}{12}, \frac{4}{15}, \frac{11}{45}\right\}\right) \\
= & \phi\left(\frac{2}{5}\right)=\frac{11}{30} .
\end{aligned}
$$

Hence, we can get

$$
G(f x, g y, h z)=\frac{1}{3}<\frac{11}{30}=\phi(M(x, y, z)) .
$$

Case 4. For $x, z \in[0,(1 / 2)], y \in((1 / 2), 1]$, then we have

$$
G(f x, g y, h z)=G\left(1, \frac{5}{6}, \frac{6}{7}\right)=\frac{1}{3} \text {. }
$$

Next we divide the study in two subcases.

(a) If $y \in((1 / 2), 1)$, then we obtain

$$
\begin{aligned}
\phi(M(x, y, z)) & \\
= & \phi\left(\operatorname { m a x } \left\{G\left(\frac{4}{5}, \frac{5}{6}, 1\right), G\left(1, \frac{4}{5}, \frac{4}{5}\right), G\left(\frac{5}{6}, \frac{5}{6}, \frac{5}{6}\right),\right.\right. \\
& G\left(\frac{6}{7}, 1,1\right), \frac{1}{3}\left[G\left(1, \frac{5}{6}, 1\right)+G\left(\frac{4}{5}, \frac{5}{6}, 1\right)\right. \\
& \left.+G\left(\frac{4}{5}, \frac{5}{6}, \frac{6}{7}\right)\right], \\
& \frac{1}{3}\left[G\left(1, \frac{5}{6}, 1\right)+G\left(1, \frac{5}{6}, \frac{6}{7}\right)\right. \\
= & \phi\left(\max \left\{\frac{2}{5}, \frac{2}{5}, 0, \frac{2}{7}, \frac{89}{315}, \frac{82}{315}\right\}\right) \\
= & \phi\left(\frac{2}{5}\right)=\frac{11}{30} .
\end{aligned}
$$

Thus we have

$$
G(f x, g y, h z)=\frac{1}{3}<\frac{11}{30}=\phi(M(x, y, z)) .
$$


(b) If $y=1$, then we can get

$$
\begin{aligned}
& \phi(M(x, y, z)) \\
&=\phi\left(\operatorname { m a x } \left\{G\left(\frac{4}{5}, 0,1\right), G\left(1, \frac{4}{5}, \frac{4}{5}\right), G\left(\frac{5}{6}, 0,0\right),\right.\right. \\
& G\left(\frac{6}{7}, 1,1\right), \frac{1}{3}\left[G(1,0,1)+G\left(\frac{4}{5}, \frac{5}{6}, 1\right)\right. \\
&+\frac{1}{3}\left[G\left(\frac{4}{5}, 0, \frac{6}{7}\right)\right], \\
&\left.\left.\left.\quad+G\left(\frac{4}{5}, \frac{5}{6}, \frac{6}{7}\right)\right]\right\}\right)+G\left(1,0, \frac{6}{7}\right) \\
&=\phi\left(\max \left\{2, \frac{2}{5}, \frac{5}{3}, \frac{2}{7}, \frac{142}{105}, \frac{77}{315}\right\}\right) \\
&=\phi(2)=\frac{11}{6} .
\end{aligned}
$$

So, we have

$$
G(f x, g y, h z)=\frac{1}{3}<\frac{11}{6}=\phi(M(x, y, z)) .
$$

Case 5. For $x \in((1 / 2), 1], y, z \in[0,(1 / 2)]$, then we have

$$
G(f x, g y, h z)=G\left(\frac{5}{6}, \frac{7}{8}, \frac{6}{7}\right)=\frac{1}{12} .
$$

Next we divide the study in two subcases.

(a) If $x \in((1 / 2), 1)$, then we can get

$$
\begin{aligned}
& \phi(M(x, y, z)) \\
& =\phi\left(\operatorname { m a x } \left\{G\left(\frac{5}{6}, 1,1\right), G\left(\frac{5}{6}, \frac{5}{6}, \frac{5}{6}\right), G\left(\frac{7}{8}, 1,1\right),\right.\right. \\
& G\left(\frac{6}{7}, 1,1\right), \frac{1}{3}\left[G\left(\frac{5}{6}, 1,1\right)+G\left(\frac{5}{6}, \frac{7}{8}, 1\right)\right. \\
& +\frac{1}{3}\left[G\left(\frac{5}{6}, \frac{7}{6}, 1, \frac{6}{7}\right)\right], \\
& \left.\left.\left.+\phi\left(\frac{5}{6}, \frac{5}{6}, \frac{6}{7}\right)\right]\right\}\right) \\
& =\phi\left(\frac{1}{3}\right)=\frac{11}{36} .
\end{aligned}
$$

Thus we have

$$
G(f x, g y, h z)=\frac{1}{12}<\frac{11}{36}=\phi(M(x, y, z)) .
$$

(b) If $x=1$, then we obtain

$$
\begin{aligned}
\phi(M(x, y, z)) & \\
= & \phi\left(\operatorname { m a x } \left\{G\left(\frac{6}{7}, 1,1\right), G\left(\frac{5}{6}, \frac{6}{7}, \frac{6}{7}\right), G\left(\frac{7}{8}, 1,1\right),\right.\right. \\
& G\left(\frac{6}{7}, 1,1\right), \frac{1}{3}\left[G\left(\frac{5}{6}, 1,1\right)+G\left(\frac{6}{7}, \frac{7}{8}, 1\right)\right. \\
& \left.+G\left(\frac{6}{7}, 1, \frac{6}{7}\right)\right], \\
& \frac{1}{3}\left[G\left(\frac{5}{6}, \frac{7}{8}, 1\right)+G\left(\frac{5}{6}, 1, \frac{6}{7}\right)\right. \\
= & \phi\left(\max \left\{\frac{2}{7}, \frac{1}{21}, \frac{1}{4}, \frac{2}{7}, \frac{19}{63}, \frac{59}{252}\right\}\right) \\
= & \phi\left(\frac{19}{63}\right)=\frac{209}{756} .
\end{aligned}
$$

Hence we get

$$
G(f x, g y, h z)=\frac{1}{12}<\frac{209}{756}=\phi(M(x, y, z)) .
$$

Case 6. For $x \in[0,(1 / 2)], y \in((1 / 2), 1]$, and $z \in((1 / 2), 1]$, then we have

$$
G(f x, g y, h z)=G\left(1, \frac{5}{6}, \frac{5}{6}\right)=\frac{1}{3} .
$$

Next we divide the study in four subcases.

(a) If $y \in((1 / 2), 1)$ and $z \in((1 / 2), 1)$, then we have

$$
\begin{aligned}
\phi & (M(x, y, z)) \\
= & \phi\left(\operatorname { m a x } \left\{G\left(\frac{4}{5}, \frac{5}{6}, \frac{5}{6}\right), G\left(1, \frac{4}{5}, \frac{4}{5}\right), G\left(\frac{5}{6}, \frac{5}{6}, \frac{5}{6}\right),\right.\right. \\
& G\left(\frac{5}{6}, \frac{5}{6}, \frac{5}{6}\right), \frac{1}{3}\left[G\left(1, \frac{5}{6}, \frac{5}{6}\right)+G\left(\frac{4}{5}, \frac{5}{6}, \frac{5}{6}\right)\right. \\
& \left.+G\left(\frac{4}{5}, \frac{5}{6}, \frac{5}{6}\right)\right], \\
& \frac{1}{3}\left[G\left(1, \frac{5}{6}, \frac{5}{6}\right)+G\left(1, \frac{5}{6}, \frac{5}{6}\right)\right. \\
= & \phi\left(\max \left\{\frac{1}{15}, \frac{2}{5}, 0,0, \frac{7}{30}, \frac{11}{30}\right\}\right) \\
= & \phi\left(\frac{2}{5}\right)=\frac{11}{30} .
\end{aligned}
$$

Thus we have

$$
G(f x, g y, h z)=\frac{1}{3}<\frac{11}{30}=\phi(M(x, y, z)) .
$$


(b) If $y=1$ and $z \in((1 / 2), 1)$, then we have

$$
\begin{aligned}
& \phi(M(x, y, z)) \\
& =\phi\left(\operatorname { m a x } \left\{G\left(\frac{4}{5}, 0, \frac{5}{6}\right), G\left(1, \frac{4}{5}, \frac{4}{5}\right), G\left(\frac{5}{6}, 0,0\right),\right.\right. \\
& G\left(\frac{5}{6}, \frac{5}{6}, \frac{5}{6}\right), \frac{1}{3}\left[G\left(1,0, \frac{5}{6}\right)+G\left(\frac{4}{5}, \frac{5}{6}, \frac{5}{6}\right)\right. \\
& \left.+G\left(\frac{4}{5}, 0, \frac{5}{6}\right)\right], \\
& \quad \frac{1}{3}\left[G\left(1, \frac{5}{6}, \frac{5}{6}\right)+G\left(1,0, \frac{5}{6}\right)\right. \\
& \left.\left.=\phi\left(\max \left\{\frac{5}{3}, \frac{2}{5}, \frac{5}{3}, 0, \frac{5}{6}, \frac{5}{6}, \frac{4}{5}\right)\right]\right\}\right) \\
& =\phi\left(\frac{5}{3}\right)=\frac{55}{36} .
\end{aligned}
$$

Therefore, we can get

$$
G(f x, g y, h z)=\frac{1}{3}<\frac{55}{36}=\phi(M(x, y, z))
$$

(c) If $y \in((1 / 2), 1)$ and $z=1$, then we get

$$
\begin{aligned}
& \phi(M(x, y, z)) \\
& =\phi\left(\operatorname { m a x } \left\{G\left(\frac{4}{5}, \frac{5}{6}, \frac{7}{8}\right), G\left(1, \frac{4}{5}, \frac{4}{5}\right), G\left(\frac{5}{6}, \frac{5}{6}, \frac{5}{6}\right),\right.\right. \\
& G\left(\frac{5}{6}, \frac{7}{8}, \frac{7}{8}\right), \frac{1}{3}\left[G\left(1, \frac{5}{6}, \frac{7}{8}\right)+G\left(\frac{4}{5}, \frac{5}{6}, \frac{7}{8}\right)\right. \\
& \left.+G\left(\frac{4}{5}, \frac{5}{6}, \frac{5}{6}\right)\right], \\
& \frac{1}{3}\left[G\left(1, \frac{5}{6}, \frac{7}{8}\right)+G\left(1, \frac{5}{6}, \frac{5}{6}\right)\right. \\
& \left.\left.=\phi\left(\max \left\{\frac{3}{20}, \frac{2}{5}, 0, \frac{1}{5}, \frac{5}{6}, \frac{5}{6}\right)\right]\right\}\right) \\
& =\phi\left(\frac{2}{5}\right)=\frac{11}{30} .
\end{aligned}
$$

Therefore, we can get

$$
G(f x, g y, h z)=\frac{1}{3}<\frac{11}{30}=\phi(M(x, y, z))
$$

(d) If $y=1$ and $z=1$, then we have

$$
\begin{aligned}
& \phi(M(x, y, z)) \\
&=\phi\left(\operatorname { m a x } \left\{G\left(\frac{4}{5}, 0, \frac{7}{8}\right), G\left(1, \frac{4}{5}, \frac{4}{5}\right), G\left(\frac{5}{6}, 0,0\right),\right.\right. \\
& G\left(\frac{5}{6}, \frac{7}{8}, \frac{7}{8}\right), \frac{1}{3}\left[G\left(1,0, \frac{7}{8}\right)+G\left(\frac{4}{5}, \frac{5}{6}, \frac{7}{8}\right)\right. \\
&\left.+G\left(\frac{4}{5}, 0, \frac{5}{6}\right)\right], \\
& \frac{1}{3}\left[G\left(1, \frac{5}{6}, \frac{7}{8}\right)+G\left(1,0, \frac{5}{6}\right)\right. \\
&\left.\left.\left.+\quad+G\left(\frac{4}{5}, \frac{5}{6}, \frac{5}{6}\right)\right]\right\}\right) \\
&=\phi\left(\max \left\{\frac{7}{4}, \frac{2}{5}, \frac{5}{3}, \frac{1}{12}, \frac{229}{180}, \frac{4}{5}\right\}\right) \\
& \phi\left(\frac{7}{4}\right)=\frac{77}{48} .
\end{aligned}
$$

Thus, we can get

$$
G(f x, g y, h z)=\frac{1}{3}<\frac{77}{48}=\phi(M(x, y, z)) .
$$

Case 7. For $x \in((1 / 2), 1], y \in[0,(1 / 2)]$, and $z \in((1 / 2), 1]$, then we have

$$
G(f x, g y, h z)=G\left(\frac{5}{6}, \frac{7}{8}, \frac{5}{6}\right)=\frac{1}{12} .
$$

Next we divide the study in four subcases.

(a) If $x \in((1 / 2), 1)$ and $z \in((1 / 2), 1)$, then we have

$$
\begin{aligned}
& \phi(M(x, y, z)) \\
& =\phi\left(\operatorname { m a x } \left\{G\left(\frac{5}{6}, 1, \frac{5}{6}\right), G\left(\frac{5}{6}, \frac{5}{6}, \frac{5}{6}\right), G\left(\frac{7}{8}, 1,1\right),\right.\right. \\
& G\left(\frac{5}{6}, \frac{5}{6}, \frac{5}{6}\right), \frac{1}{3}\left[G\left(\frac{5}{6}, 1, \frac{5}{6}\right)+G\left(\frac{5}{6}, \frac{7}{8}, \frac{5}{6}\right)\right. \\
& \left.+G\left(\frac{5}{6}, 1, \frac{5}{6}\right)\right], \\
& \quad \frac{1}{3}\left[G\left(\frac{5}{6}, \frac{7}{8}, \frac{5}{6}\right)+G\left(\frac{5}{6}, 1, \frac{5}{6}\right)\right. \\
& \left.\left.\left.+G\left(\frac{5}{6}, \frac{7}{8}, \frac{5}{6}\right)\right]\right\}\right) \\
& =\phi\left(\frac{1}{3}\right)=\frac{11}{36} .
\end{aligned}
$$


Thus, we can get

$$
G(f x, g y, h z)=\frac{1}{12}<\frac{11}{36}=\phi(M(x, y, z)) .
$$

(b) If $x=1$ and $z \in((1 / 2), 1)$, then we have

$$
\begin{aligned}
\phi(M(x, y, z)) & \phi\left(\operatorname { m a x } \left\{G\left(\frac{6}{7}, 1, \frac{5}{6}\right), G\left(\frac{5}{6}, \frac{6}{7}, \frac{6}{7}\right), G\left(\frac{7}{8}, 1,1\right),\right.\right. \\
& G\left(\frac{5}{6}, \frac{5}{6}, \frac{5}{6}\right), \frac{1}{3}\left[G\left(\frac{5}{6}, 1, \frac{5}{6}\right)+G\left(\frac{6}{7}, \frac{7}{8}, \frac{5}{6}\right)\right. \\
& \left.+G\left(\frac{6}{7}, 1, \frac{5}{6}\right)\right], \\
& \frac{1}{3}\left[G\left(\frac{5}{6}, \frac{7}{8}, \frac{5}{6}\right)+G\left(\frac{5}{6}, 1, \frac{5}{6}\right)\right. \\
= & \phi\left(\max \left\{\frac{1}{3}, \frac{1}{21}, \frac{1}{4}, 0, \frac{1}{4}, \frac{1}{6}\right\}\right) \\
= & \phi\left(\frac{1}{3}\right)=\frac{11}{36} .
\end{aligned}
$$

Hence, we obtain

$$
G(f x, g y, h z)=\frac{1}{12}<\frac{11}{36}=\phi(M(x, y, z)) .
$$

(c) If $x \in((1 / 2), 1)$ and $z=1$, then we get

$$
\begin{aligned}
& \phi(M(x, y, z)) \\
&=\phi\left(\operatorname { m a x } \left\{G\left(\frac{5}{6}, 1, \frac{7}{8}\right), G\left(\frac{5}{6}, \frac{5}{6}, \frac{5}{6}\right), G\left(\frac{7}{8}, 1,1\right),\right.\right. \\
& G\left(\frac{5}{6}, \frac{7}{8}, \frac{7}{8}\right), \frac{1}{3}\left[G\left(\frac{5}{6}, 1, \frac{7}{8}\right)+G\left(\frac{5}{6}, \frac{7}{8}, \frac{7}{8}\right)\right. \\
&\left.+G\left(\frac{5}{6}, 1, \frac{5}{6}\right)\right], \\
& \quad \frac{1}{3}\left[G\left(\frac{5}{6}, \frac{7}{8}, \frac{7}{8}\right)+G\left(\frac{5}{6}, 1, \frac{5}{6}\right)\right. \\
&\left.\left.\left.=\phi\left(\max \left\{\frac{1}{3}, 0, \frac{1}{4}, \frac{1}{12}, \frac{1}{8}, \frac{1}{6}, \frac{1}{6}\right\}\right)\right]\right\}\right) \\
&=\phi\left(\frac{1}{3}\right)=\frac{11}{36} .
\end{aligned}
$$

Therefore, we have

$$
G(f x, g y, h z)=\frac{1}{12}<\frac{11}{36}=\phi(M(x, y, z)) .
$$

(d) If $x=1$ and $z=1$, then we have

$\phi(M(x, y, z))$

$$
\begin{aligned}
& =\phi\left(\operatorname { m a x } \left\{G\left(\frac{6}{7}, 1, \frac{7}{8}\right), G\left(\frac{5}{6}, \frac{6}{7}, \frac{6}{7}\right), G\left(\frac{7}{8}, 1,1\right),\right.\right. \\
& G\left(\frac{5}{6}, \frac{7}{8}, \frac{7}{8}\right), \frac{1}{3}\left[G\left(\frac{5}{6}, 1, \frac{7}{8}\right)+G\left(\frac{6}{7}, \frac{7}{8}, \frac{7}{8}\right)\right. \\
& \left.+G\left(\frac{6}{7}, 1, \frac{5}{6}\right)\right], \\
& \frac{1}{3}\left[G\left(\frac{5}{6}, \frac{7}{8}, \frac{7}{8}\right)+G\left(\frac{5}{6}, 1, \frac{5}{6}\right)\right. \\
& =\phi\left(\max \left\{\frac{2}{7}, \frac{1}{12}, \frac{1}{4}, \frac{1}{12}, \frac{59}{252}, \frac{1}{6}\right\}\right) \\
& =\phi\left(\frac{2}{7}\right)=\frac{11}{42} .
\end{aligned}
$$

Thus, we obtain

$$
G(f x, g y, h z)=\frac{1}{12}<\frac{11}{42}=\phi(M(x, y, z)) .
$$

Case 8. For $x \in((1 / 2), 1], y \in((1 / 2), 1]$, and $z \in[0,(1 / 2)]$, then we have

$$
G(f x, g y, h z)=G\left(\frac{5}{6}, \frac{5}{6}, \frac{6}{7}\right)=\frac{1}{21} .
$$

Next we divide the study in four subcases.

(a) If $x \in((1 / 2), 1)$ and $y \in((1 / 2), 1)$, then we get

$$
\begin{aligned}
\phi(M(x, y, z)) & \phi\left(\operatorname { m a x } \left\{G\left(\frac{5}{6}, \frac{5}{6}, 1\right), G\left(\frac{5}{6}, \frac{5}{6}, \frac{5}{6}\right), G\left(\frac{5}{6}, \frac{5}{6}, \frac{5}{6}\right),\right.\right. \\
& G\left(\frac{6}{7}, 1,1\right), \frac{1}{3}\left[G\left(\frac{5}{6}, \frac{5}{6}, 1\right)+G\left(\frac{5}{6}, \frac{5}{6}, 1\right)\right. \\
& \left.+G\left(\frac{5}{6}, \frac{5}{6}, \frac{6}{7}\right)\right], \\
& \frac{1}{3}\left[G\left(\frac{5}{6}, \frac{5}{6}, 1\right)+G\left(\frac{5}{6}, \frac{5}{6}, \frac{6}{7}\right)\right. \\
= & \phi\left(\max \left\{\frac{1}{3}, 0,0, \frac{2}{7}, \frac{5}{21}, \frac{1}{7}\right\}\right) \\
= & \phi\left(\frac{1}{3}\right)=\frac{11}{36} .
\end{aligned}
$$

Thus, we obtain

$$
G(f x, g y, h z)=\frac{1}{21}<\frac{11}{36}=\phi(M(x, y, z)) .
$$


(b) If $x=1$ and $y \in((1 / 2), 1)$, then we have

$$
\begin{aligned}
& \phi(M(x, y, z)) \\
&=\phi\left(\operatorname { m a x } \left\{G\left(\frac{6}{7}, \frac{5}{6}, 1\right), G\left(\frac{5}{6}, \frac{6}{7}, \frac{6}{7}\right), G\left(\frac{5}{6}, \frac{5}{6}, \frac{5}{6}\right),\right.\right. \\
& G\left(\frac{6}{7}, 1,1\right), \frac{1}{3}\left[G\left(\frac{5}{6}, \frac{5}{6}, 1\right)+G\left(\frac{6}{7}, \frac{5}{6}, 1\right)\right. \\
&\left.+G\left(\frac{6}{7}, \frac{5}{6}, \frac{6}{7}\right)\right], \\
& \quad \frac{1}{3}\left[G\left(\frac{5}{6}, \frac{5}{6}, 1\right)+G\left(\frac{5}{6}, \frac{5}{6}, \frac{6}{7}\right)\right. \\
&\left.\left.=\phi\left(\max \left\{\frac{1}{7}, \frac{1}{21}, 0, \frac{2}{6}, \frac{6}{7}\right)\right]\right\}\right) \\
&=\phi\left(\frac{1}{21}\right)=\frac{11}{36} .
\end{aligned}
$$

Thus, we obtain

$$
G(f x, g y, h z)=\frac{1}{21}<\frac{11}{36}=\phi(M(x, y, z)) .
$$

(c) If $x \in((1 / 2), 1)$ and $y=1$, then we obtain

$$
\begin{gathered}
\phi(M(x, y, z)) \\
=\phi\left(\operatorname { m a x } \left\{G\left(\frac{5}{6}, 0,1\right), G\left(\frac{5}{6}, \frac{5}{6}, \frac{5}{6}\right), G\left(\frac{5}{6}, 0,0\right),\right.\right. \\
G\left(\frac{6}{7}, 1,1\right), \frac{1}{3}\left[G\left(\frac{5}{6}, 0,1\right)+G\left(\frac{5}{6}, \frac{5}{6}, 1\right)\right. \\
\left.+G\left(\frac{5}{6}, 0, \frac{6}{7}\right)\right], \\
\frac{1}{3}\left[G\left(\frac{5}{6}, \frac{5}{6}, 1\right)+G\left(\frac{5}{6}, 0, \frac{6}{7}\right)\right. \\
\left.\left.\left.+G\left(\frac{5}{6}, \frac{5}{6}, \frac{6}{7}\right)\right]\right\}\right) \\
=\phi\left(\max \left\{2,0, \frac{5}{3}, \frac{2}{7}, \frac{85}{63}, \frac{44}{63}\right\}\right) \\
=\phi(2)=\frac{11}{6} .
\end{gathered}
$$

Thus, we obtain

$$
G(f x, g y, h z)=\frac{1}{21}<\frac{11}{6}=\phi(M(x, y, z)) .
$$

(d) If $x=1$ and $y=1$, then we have

$$
\begin{gathered}
\phi(M(x, y, z)) \\
=\phi\left(\operatorname { m a x } \left\{G\left(\frac{6}{7}, 0,1\right), G\left(\frac{5}{6}, \frac{6}{7}, \frac{6}{7}\right), G\left(\frac{5}{6}, 0,0\right),\right.\right. \\
G\left(\frac{6}{7}, 1,1\right), \frac{1}{3}\left[G\left(\frac{5}{6}, 0,1\right)+G\left(\frac{6}{7}, \frac{5}{6}, 1\right)\right. \\
\left.+G\left(\frac{6}{7}, 0, \frac{6}{7}\right)\right], \\
\frac{1}{3}\left[G\left(\frac{5}{6}, \frac{5}{6}, 1\right)+G\left(\frac{5}{6}, 0, \frac{6}{7}\right)\right. \\
\left.\left.\left.+G\left(\frac{6}{7}, \frac{5}{6}, \frac{6}{7}\right)\right]\right\}\right) \\
=\phi\left(\max \left\{2, \frac{1}{21}, \frac{5}{3}, \frac{2}{7}, \frac{85}{63}, \frac{44}{63}\right\}\right) \\
=\phi(2)=\frac{11}{6} .
\end{gathered}
$$

Thus, we obtain

$$
G(f x, g y, h z)=\frac{1}{21}<\frac{11}{6}=\phi(M(x, y, z)) .
$$

Then in all the above cases, the mappings $f, g, h, R, S$, and $T$ are satisfying conditions (3) and (i) of Theorem 15 , so that all the conditions of Theorem 15 are satisfied. Moreover, 5/6 is the unique common fixed point of $f, g, h, R, S$, and $T$.

Remark 17. In this paper, we get the new common fixed point theorem using the common (E.A) property with three pairs of weakly compatible mappings. This is a new result has never been discussed by other authors.

Remark 18. If we take (1) $R=S=T$; (2) $f=g=h$; (3) $R=S=T=I$ ( $I$ is identity mapping); (4) $S=T$ and $g=h$; (5) $S=T, g=h=I$ in Theorem 15, then several new results can be obtained.

Corollary 19. Let $(X, G)$ be a $G$-metric space. Suppose mappings $f, g, h, R, S$, and $T: X \rightarrow X$ satisfy the following conditions:

$G(f x, g y, h z)$

$\leq \lambda \max \{G(R x, S y, T z), G(f x, R x, R x), G(g y, S y, S y)$,

$$
\begin{aligned}
& G(h z, T z, T z), \frac{1}{3}[G(f x, S y, T z)+G(R x, g y, T z) \\
& +G(R x, S y, h z)], \\
& \frac{1}{3}[G(f x, g y, T z)+G(f x, S y, h z) \\
& +G(R x, g y, h z)]\}
\end{aligned}
$$


for all $x, y$, and $z \in X$, where $\lambda \in[0,1)$. If one of the following conditions is satisfied, then the pairs $(f, R),(g, S)$, and $(h, T)$ have a common point of coincidence in $X$.

(i) The subspace $R X$ is closed in $X, f X \subseteq S X, g X \subseteq T X$, and two pairs of $(f, R)$ and $(g, S)$ satisfy common $(E . A)$ property.

(ii) The subspace $S X$ is closed in $X, g X \subseteq T X, h X \subseteq R X$, and two pairs of $(g, S)$ and $(h, T)$ satisfy common $(E . A)$ property.

(iii) The subspace $T X$ is closed in $X, f X \subseteq S X, h X \subseteq R X$, and two pairs of $(f, R)$ and $(h, T)$ satisfy common (E.A) property.

Moreover, if the pairs $(f, R),(g, S)$, and $(h, T)$ are weakly compatible, then $f, g, h, R, S$, and $T$ have a unique common fixed point in $X$.

Proof. Taking $\phi(t)=\lambda t, \lambda \in[0,1)$ in Theorem 15, the conclusion of Corollary 19 can be obtained from Theorem 15 immediately. This completes the proof of Corollary 19.

Remark 20. If we take (1) $R=S=T$; (2) $f=g=h$; (3) $R=S=T=I$ ( $I$ is identity mapping); (4) $S=T$ and $g=h$; (5) $S=T, g=h=I$ in Corollary 19, then several new results can be obtained.

Corollary 21. Let $(X, G)$ be a G-metric space. Suppose mappings $f, g, h, R, S$, and $T: X \rightarrow X$ satisfy the following conditions:

$$
\begin{aligned}
G(f x, g y, h z) \leq & a_{1} G(R x, S y, T z)+a_{2} G(f x, R x, R x) \\
+ & a_{3} G(g y, S y, S y)+a_{4} G(h z, T z, T z) \\
& +a_{5}[G(f x, S y, T z)+G(R x, g y, T z) \\
& +G(R x, S y, h z)] \\
+ & a_{6}[G(f x, g y, T z)+G(f x, S y, h z) \\
& +G(R x, g y, h z)]
\end{aligned}
$$

for all $x, y$, and $z \in X$, where $a_{i} \geq 0(i=1,2,3,4,5$, and 6$)$ and $0 \leq a_{1}+a_{2}+a_{3}+a_{4}+3 a_{5}+3 a_{6}<1$. If one of the following conditions is satisfied, then the pairs $(f, R),(g, S)$, and $(h, T)$ have a common point of coincidence in $X$ :

(i) the subspace $R X$ is closed in $X, f X \subseteq S X, g X \subseteq T X$, and two pairs of $(f, R)$ and $(g, S)$ satisfy common (E.A) property;

(ii) the subspace $S X$ is closed in $X, g X \subseteq T X, h X \subseteq R X$, and two pairs of $(g, S)$ and $(h, T)$ satisfy common (E.A) property;

(iii) the subspace $T X$ is closed in $X, f X \subseteq S X, h X \subseteq R X$, and two pairs of $(f, R)$ and $(h, T)$ satisfy common (E.A) property.

Moreover, if the pairs $(f, R),(g, S)$, and $(h, T)$ are weakly compatible, then $f, g, h, R, S$, and $T$ have a unique common fixed point in $X$.
Proof. Suppose that

$$
\begin{aligned}
& M(x, y, z) \\
& =\max \{G(R x, S y, T z), G(f x, R x, R x), G(g y, S y, S y), \\
& G(h z, T z, T z), \\
& \frac{1}{3}[G(f x, S y, T z)+G(R x, g y, T z) \\
& +G(R x, S y, h z)], \\
& \frac{1}{3}[G(f x, g y, T z)+G(f x, S y, h z) \\
& +G(R x, g y, h z)]\} .
\end{aligned}
$$

Then

$$
\begin{aligned}
a_{1} G & (R x, S y, T z)+a_{2} G(f x, R x, R x) \\
& +a_{3} G(g y, S y, S y)+a_{4} G(h z, T z, T z) \\
& +a_{5}[G(f x, S y, T z)+G(R x, g y, T z)+G(R x, S y, h z)] \\
& +a_{6}[G(f x, g y, T z)+G(f x, S y, h z)+G(R x, g y, h z)] \\
\leq & \left(a_{1}+a_{2}+a_{3}+a_{4}+3 a_{5}+3 a_{6}\right) M(x, y, z) .
\end{aligned}
$$

So, if condition (61) holds, then $G(f x, g y, h z) \leq\left(a_{1}+a_{2}+a_{3}+\right.$ $\left.a_{4}+3 a_{5}+3 a_{6}\right) M(x, y, z)$. Taking $\lambda=a_{1}+a_{2}+a_{3}+a_{4}+3 a_{5}+3 a_{6}$ in Corollary 19, the conclusion of Corollary 21 can be obtained from Corollary 19 immediately.

Remark 22. If we take (1) $R=S=T$; (2) $f=g=h$; (3) $R=S=T=I$ ( $I$ is identity mapping); (4) $S=T$ and $g=h$; (5) $S=T, g=h=I$ in Corollary 21, then several new results can be obtained.

\section{Acknowledgments}

The present studies are supported by the National Natural Science Foundation of China (11071169, 11271105), the Natural Science Foundation of Zhejiang Province (Y6110287, LY12A01030), and the Physical Experiment Center of Hangzhou Normal University.

\section{References}

[1] Z. Mustafa and B. Sims, "A new approach to generalized metric spaces," Journal of Nonlinear and Convex Analysis, vol. 7, no. 2, pp. 289-297, 2006.

[2] Z. Mustafa, H. Obiedat, and F. Awawdeh, "Some fixed point theorem for mapping on complete $G$-metric spaces," Fixed Point Theory and Applications, vol. 2008, Article ID 189870, 12 pages, 2008.

[3] Z. Mustafa and B. Sims, "Fixed point theorems for contractive mappings in complete G-metric spaces," Fixed Point Theory and Applications, vol. 2009, Article ID 917175, 10 pages, 2009. 
[4] Z. Mustafa, W. Shatanawi, and M. Bataineh, "Existence of fixed point results in G-metric spaces," International Journal of Mathematics and Mathematical Sciences, vol. 2009, Article ID 283028, 10 pages, 2009.

[5] Z. Mustafa, M. Khandagji, and W. Shatanawi, "Fixed point results on complete G-metric spaces," Studia Scientiarum Mathematicarum Hungarica, vol. 48, no. 3, pp. 304-319, 2011.

[6] H. Obiedat and Z. Mustafa, "Fixed point results on a nonsymmetric G-metric spaces," Jordan Journal of Mathematics and Statistics, vol. 3, no. 2, pp. 65-79, 2010.

[7] H. Aydi, W. Shatanawi, and C. Vetro, "On generalized weak $G$-contraction mapping in G-metric spaces," Computers \& Mathematics with Applications, vol. 62, no. 11, pp. 4223-4229, 2011.

[8] H. Aydi, "A fixed point result involving a generalized weakly contractive condition in G-metric spaces," Bulletin of Mathematical Analysis and Applications, vol. 3, no. 4, pp. 180-188, 2011.

[9] Lj. Gajić and M. Stojaković, "On Ćirić generalization of mappings with a contractive iterate at a point in G-metric spaces," Applied Mathematics and Computation, vol. 219, no. 1, pp. 435441, 2012.

[10] S. Zhou and F. Gu, "Some new fixed points in G-metric spaces," Journal of Hangzhou Normal University, vol. 11, no. 1, pp. 47-50, 2012.

[11] W. Shatanawi, "Fixed point theory for contractive mappings satisfying $\Phi$-maps in G-metric spaces," Fixed Point Theory and Applications, vol. 2010, Article ID 181650, 9 pages, 2010.

[12] R. Chugh, T. Kadian, A. Rani, and B. E. Rhoades, "Property $P$ in G-metric spaces," Fixed Point Theory and Applications, vol. 2010, Article ID 401684, 12 pages, 2010.

[13] M. Abbas and B. E. Rhoades, "Common fixed point results for noncommuting mappings without continuity in generalized metric spaces," Applied Mathematics and Computation, vol. 215, no. 1, pp. 262-269, 2009.

[14] M. Abbas, T. Nazir, and R. Saadati, "Common fixed point results for three maps in generalized metric space," Advances in Difference Equations, vol. 2011, article 49, 2011.

[15] M. Abbas, T. Nazir, and S. Radenović, "Some periodic point results in generalized metric spaces," Applied Mathematics and Computation, vol. 217, no. 8, pp. 4094-4099, 2010.

[16] M. Abbas, S. H. Khan, and T. Nazir, "Common fixed points of $R$-weakly commuting maps in generalized metric spaces," Fixed Point Theory and Applications, vol. 2011, article 41, 2011.

[17] R. K. Vats, S. Kumar, and V. Sihag, "Some common fixed point theorems for compatible mappings of type $(A)$ in complete $G$ metric space," Advances in Fuzzy Mathematics, vol. 6, no. 1, pp. $27-38,2011$.

[18] M. Abbas, T. Nazir, and P. Vetro, "Common fixed point results for three maps in G-metric spaces," Filomat, vol. 25, no. 4, pp. $1-17,2011$.

[19] S. Manro, S. Kumar, and S. S. Bhatia, " $R$-weakly commuting maps in G-metric spaces," Fasciculi Mathematici, no. 47, pp. 1117, 2011.

[20] F. Gu, "Common fixed point theorems for six mappings in generalized metric spaces," Abstract and Applied Analysis, vol. 2012, Article ID 379212, 21 pages, 2012.

[21] F. Gu and H. Ye, "Common fixed point theorems of Altman integral type mappings in G-metric spaces," Abstract and Applied Analysis, vol. 2012, Article ID 630457, 13 pages, 2012.

[22] H. Ye and F. Gu, "Common fixed point theorems for a class of twice power type contraction maps in G-metric spaces,"
Abstract and Applied Analysis, vol. 2012, Article ID 736214, 19 pages, 2012.

[23] Y. Yin and F. Gu, "Common fixed point theorem about four mappings in G-metric spaces," Journal of Hangzhou Normal University, vol. 11, no. 6, pp. 511-515, 2012.

[24] A. Kaewcharoen, "Common fixed point theorems for contractive mappings satisfying $\Phi$-maps in G-metric spaces," Banach Journal of Mathematical Analysis, vol. 6, no. 1, pp. 101-111, 2012.

[25] N. Tahat, H. Aydi, E. Karapinar, and W. Shatanawi, "Common fixed points for singlevalued and multi-valued maps satisfying a generalized contraction in G-metric spaces," Fixed Point Theory and Applications, vol. 2012, 48 pages, 2012.

[26] Z. Mustafa, "Some new common fixed point theorems under strict contractive conditions in G-metric spaces," Journal of Applied Mathematics, vol. 2012, Article ID 248937, 21 pages, 2012.

[27] A. Kaewcharoen, "Common fixed points for four mappings in G-metric spaces," International Journal of Mathematical Analysis, vol. 6, no. 45-48, pp. 2345-2356, 2012.

[28] V. Popa and A.-M. Patriciu, "A general fixed point theorem for pairs of weakly compatible mappings in G-metric spaces," Journal of Nonlinear Science and its Applications, vol. 5, no. 2, pp. 151-160, 2012.

[29] Z. Mustafa, "Common fixed points of weakly compatible mappings in G-metric spaces," Applied Mathematical Sciences, vol. 6, no. 92, pp. 4589-4600, 2012.

[30] M. Gugnani, M. Aggarwal, and R. Chugh, "Common fixed point results in $G$-metric spaces and applications," International Journal of Computer Applications, vol. 43, no. 11, pp. 38-42, 2012.

[31] H. Aydi, "A common fixed point of integral type contraction in generalized metric spaces," Journal of Advanced Mathematical Studies, vol. 5, no. 1, pp. 111-117, 2012.

[32] K. P. R. Rao, K. Bhanu Lakshmi, Z. Mustafa, and V. C. C. Raju, "Fixed and related fixed point theorems for three maps in Gmetric spaces," Journal of Advanced Studies in Topology, vol. 3, no. 4, pp. 12-19, 2012.

[33] M. Abbas, T. Nazir, and D. Dorić, "Common fixed point of mappings satisfying (E.A) property in generalized metric spaces," Applied Mathematics and Computation, vol. 218, no. 14, pp. 7665-7670, 2012.

[34] Z. Mustafa, H. Aydi, and E. Karapınar, "On common fixed points in G-metric spaces using (E.A) property," Computers \& Mathematics with Applications, vol. 64, no. 6, pp. 1944-1956, 2012.

[35] W. Long, M. Abbas, T. Nazir, and S. Radenović, "Common fixed point for two pairs of mappings satisfying (E.A) property in generalized metric spaces," Abstract and Applied Analysis, vol. 2012, Article ID 394830, 15 pages, 2012.

[36] G. Jungck and B. E. Rhoades, "Fixed points for set valued functions without continuity," Indian Journal of Pure and Applied Mathematics, vol. 29, no. 3, pp. 227-238, 1998. 


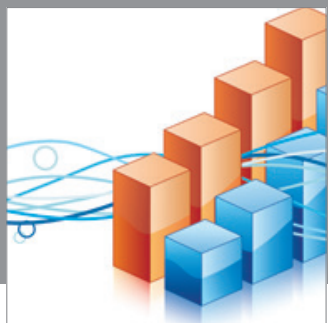

Advances in

Operations Research

mansans

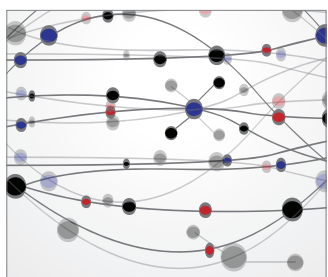

The Scientific World Journal
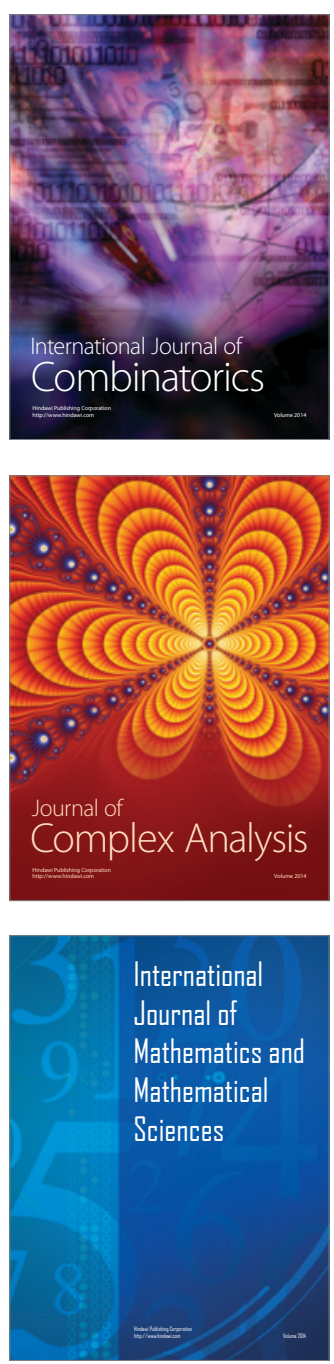
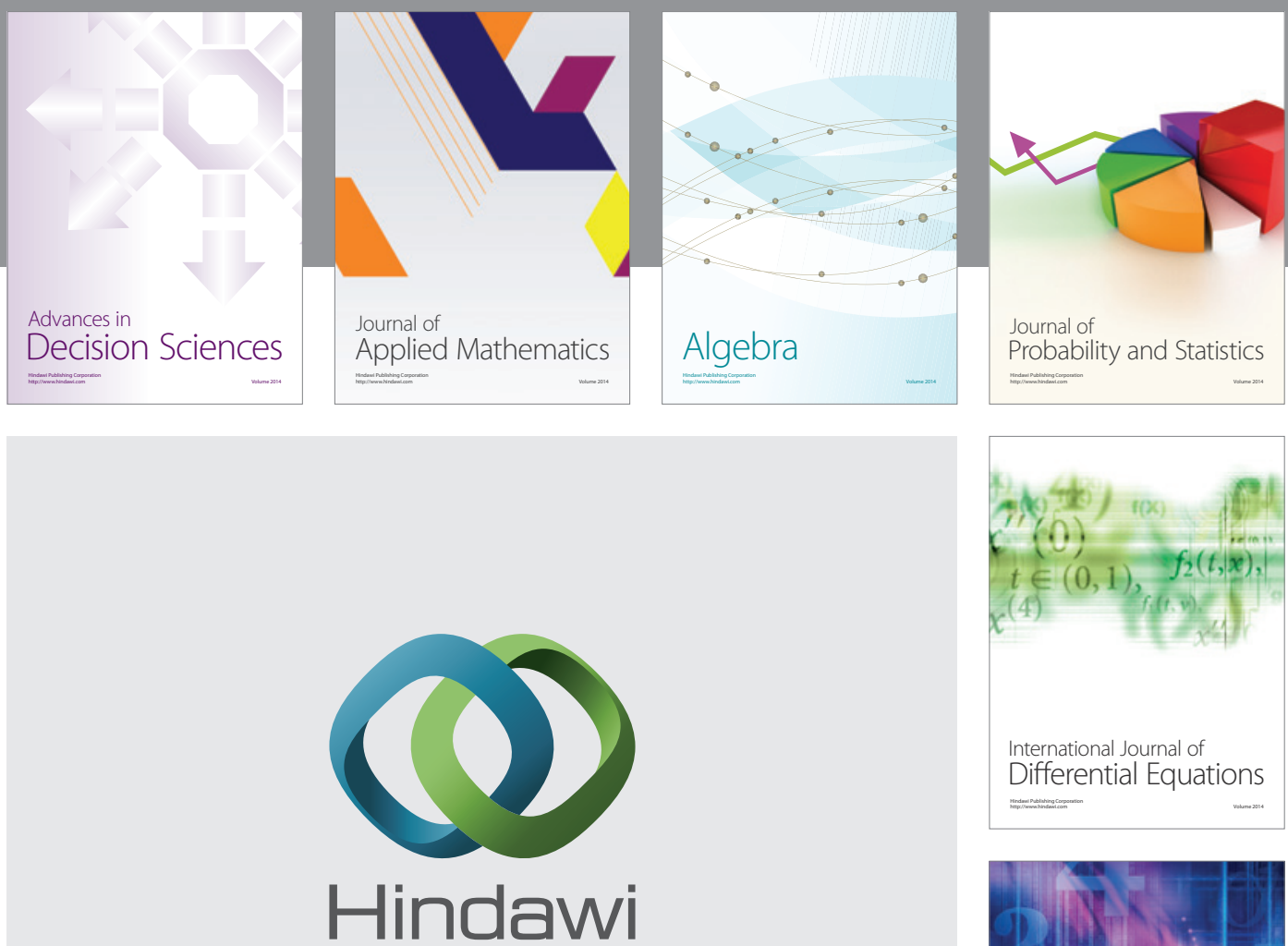

Submit your manuscripts at http://www.hindawi.com
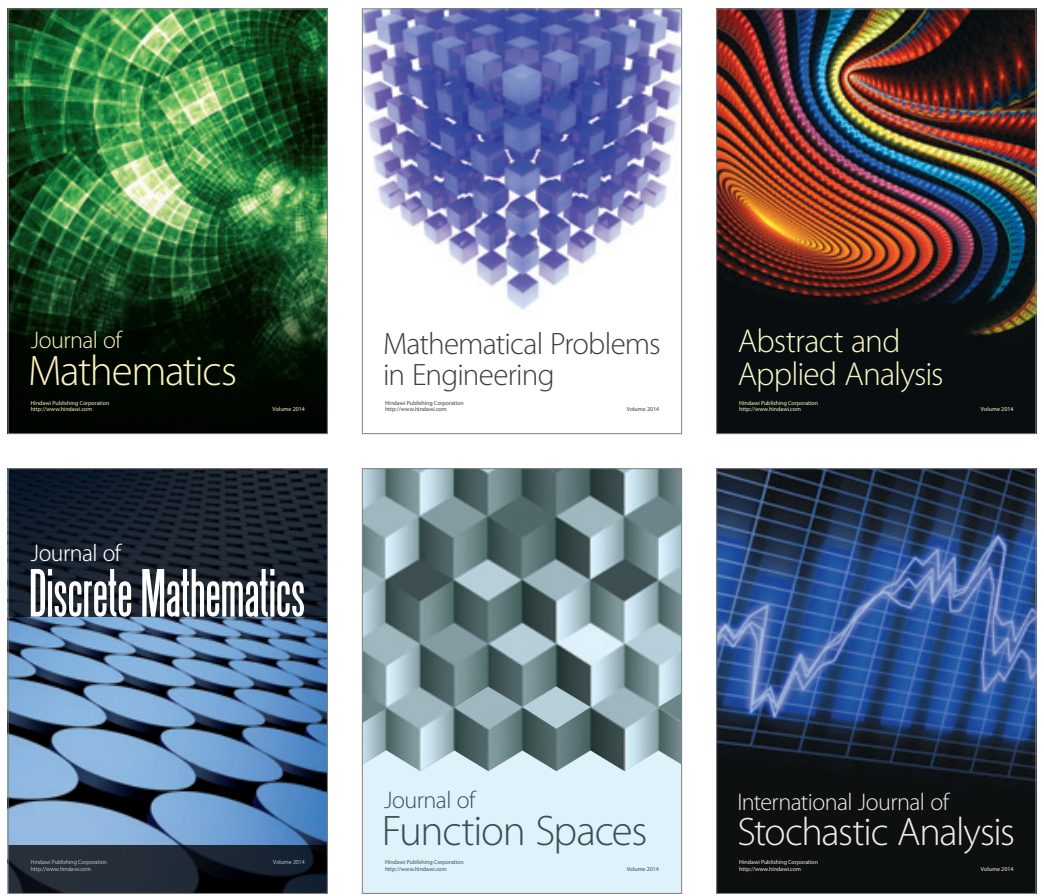

Journal of

Function Spaces

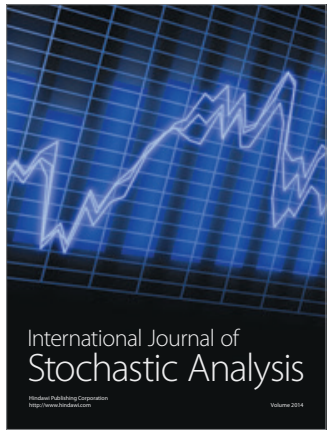

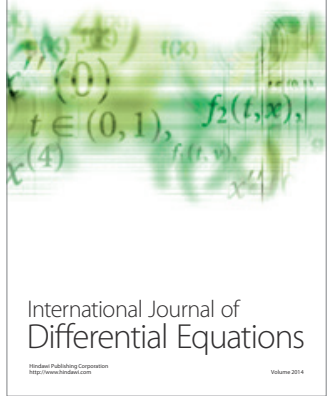
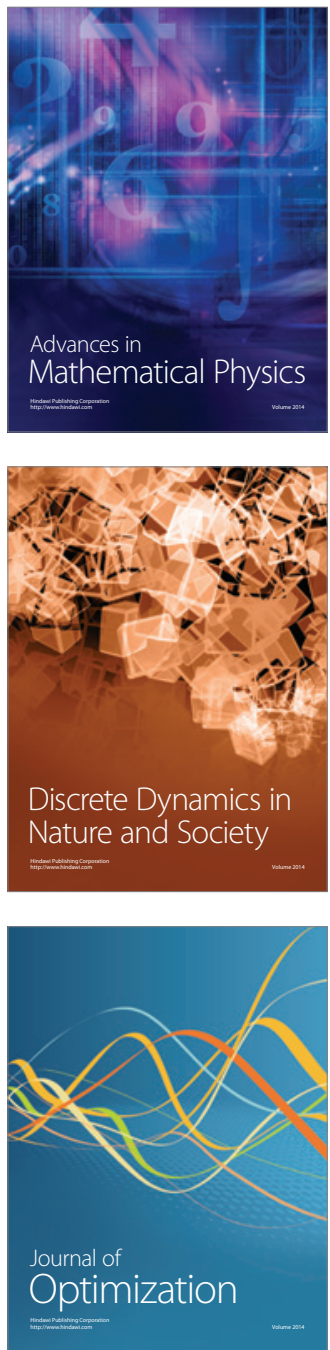